DOI: https://doi.org/10.46296/yc.v4i7.0047

\title{
DISEÑO DE UN SISTEMA DE GESTIÓN DE CALIDAD PEDAGÓGICA ENFOCADO A LA NORMA ISO 9001-2015 PARA EL COLEGIO "RUBÉN DARÍO" DEL CANTÓN MANTA.
}

\section{DESIGN OF A PEDAGOGICAL QUALITY MANAGEMENT SYSTEM FOCUSED ON THE ISO 9001-2015 STANDARD FOR THE "RUBEN DARIO" SCHOOL OF CANTON MANTA.}

\author{
Loor-Burgos Mayra Patricia ${ }^{1 *}$; Henríquez-Coronel María Angélica ${ }^{2}$ \\ ${ }^{1}$ Estudiante de la Maestría en Gerencia Calidad e Innovación de la Universidad \\ Técnica de Manabí, UTM. Portoviejo, Ecuador. \\ ${ }^{2}$ Docente Investigadora de la Facultad de Filosofía, Letras y Ciencias de la Educación \\ de la Universidad Técnica de Manabí, UTM. Portoviejo, Ecuador.
}

${ }^{\star}$ Correo: patricialoorpattyloor@hotmail.com

\begin{abstract}
Resumen
La presente investigación tiene como objetivo proponer el uso de un sistema de gestión de calidad basado en la norma ISO 9001:2015@ como herramienta que puede contribuir a la mejora académica en el colegio Rubén Darío de la ciudad Manta, Ecuador, lo que permitió el establecimiento de los objetivos específicos, mismos que fueron alcanzados a través de la investigación de campo mediante la aplicación de técnicas como encuestas a los docentes, personal administrativo y autoridad. Los resultados de la investigación de campo fueron sometidos al análisis y debate teórico bajo los lineamientos y directrices de la norma ISO 9001 2015 , luego de lo cual se establecieron las conclusiones respectivas. Por otra parte, se procedió a realizar el proceso de validación de resultados, llegándose a la conclusión que, en la Unidad Educativa Rubén Darío, existen algunas deficiencias, las políticas y objetivos, una visión y misión que no están claramente definidas y son desconocidas por una parte importante del personal existiendo procesos inadecuados que afectan el área administrativa. Mediante el uso de las técnicas de investigación, se realizó encuesta al talento humano de la institución, con la finalidad de realizar un estudio referente a la importancia de la implementación de un sistema de gestión de calidad. La conclusión, orientó la determinación de recomendaciones tales como ejecutar la propuesta del diseño de un Sistema de Gestión de Calidad basado en la Norma ISO 9001:2015@ en la Unidad Educativa Rubén Darío, para optimizar sus procesos, permitiendo brindar un servicio eficiente con calidad y calidez a la comunidad.
\end{abstract}

Palabras Claves: Desarrollo Institucional, Gestión de calidad, Instituciones Educativas, Norma ISO 9001-2015.

\begin{abstract}
The present research aims to propose the use of a quality management system based on the ISO 9001: 2015 standard as a tool that contributes to academic improvement at the Rubén Darío school, which allowed the establishment of specific objectives, themselves which were achieved through field research through the application of techniques such as surveys of teachers, administrative staff and authority, The results of the field research were subjected to analysis and theoretical debate under the guidelines and guidelines of the ISO 9001 standard -2015, after which the respective conclusions were established. On the other hand, the results validation process was carried out, reaching the conclusion that In the Rubén Darío Educational Unit, some deficiencies were found, such as, for example, it does not have a quality policy, nor quality objectives, a organization chart and there is also reprocessing that affects the administrative area, a vision and mission not very clear in addition to the ignorance of these in the staff. Through the
\end{abstract}

Información del manuscrito:

Fecha de recepción: 05 de mayo de 2020.

Fecha de aceptación: 09 de julio de 2020.

Fecha de publicación: 10 de julio de 2020. 
use of research techniques, a survey was carried out on the institution's human talent, in order to carry out a study regarding the importance of the implementation of a quality management system. The conclusion guided the determination of recommendations such as executing the proposal for the design of a Quality Management System based on the ISO 9001: 2015 Standard in the Rubén Darío Educational Unit, to optimize its processes, allowing to provide an efficient service with quality and warmth to the community.

Keywords: Quality management, ISO 9001-2015 Standard, Institutional Development, Educational Institutions.

\section{Introducción}

Las instituciones educativas tienen como objetivo ofrecer una educación de calidad para el beneficio de la sociedad y la formación del futuro del país. Es por esto que el presente trabajo de investigación propone la implementación de un Sistema de Calidad ISO 9001:2015๑. La norma describe diversos requisitos $y$ procedimientos a seguir para llegar a la calidad total y al mismo tiempo, mejorar la imagen y prestigio del colegio. El Sistema de calidad ISO 9001:2015@ ayudará al colegio a crear valor agregado, comprender las necesidades actuales y futuras usando procedimientos y mejora continua. Pondrá a disposición de la institución todas las herramientas y lineamientos necesarios para ser más competitiva.

La gestión es un elemento determinante de la calidad del desempeño de las instituciones educativas (López, 2010), sobre todo en la medida que se incrementa la descentralización de los procesos de decisión en los sistemas educacionales, incide en el clima organizacional, en las formas de liderazgo y conducción institucionales, en el aprovechamiento óptimo de los recursos humanos y del tiempo, en la planificación de tareas, la distribución del trabajo y su productividad sobre todo en instituciones educativas donde el esfuerzo debe verse revertido en el proceso de enseñanza aprendizaje, a través de la organización y planificación.

De acuerdo con la información proporcionada por la Secretaría del plantel, la institución cuenta con los niveles educativos correspondientes a Inicial, Preparatoria, Básica Inferior, Básica Media, Básica Superior y Bachillerato, y cuenta con una población estudiantil de 180 estudiantes, 17 docentes y 4 miembros del personal administrativo. Como indica Areche 
(2016): "Cada compañía debe encontrar el plan de juego más adecuado para sobrevivir y crecer a largo plazo, considerando su situación específica, sus oportunidades, sus objetivos y sus recursos".

La noción de calidad nos remite a la idea de excelencia, tanto en los procesos como de los productos 0 servicios que una organización proporciona, y evoca facetas tales como buen clima de trabajo, posición destacada en el sector, buen funcionamiento organizativo, alta consideración tanto interna como externa, elevada rentabilidad económica y social, pues como nos dice López, "calidad es la satisfacción de las necesidades y expectativas de los clientes" (2003) Para Pérez (2000), al buscar la calidad, debemos partir de la idea de que cualquier cosa puede hacerse mejor y de que toda mejora es importante, aunque a primera vista parezca insignificante. Se debe tomar conciencia de que la calidad en educación se trabaja más en el proceso que en el producto.

Tradicionalmente en Ecuador la administración educativa ha estado a cargo de personas que no cumplen con los estándares especificado para el desempeño eficiente de su función, pues en muchas ocasiones no son del campo docente. La falta de conciencia de que la administración de la educación es una actividad especializada, que genera una mentalidad burocrática en algunos sectores, serán trabas que deben ser superadas si se quiere modernizar el sistema educativo. (Pérez, R, 2000) En la actualidad el Ministerio de Educación ha implantado un sistema de evaluación para todas las autoridades de los planteles educativos, y se realizan concursos de mérito y oposición, donde los candidatos demuestran las capacidades que poseen para realizar una eficaz gestión administrativa de las instituciones educativas, y de este modo, proveerlas de líderes que tengan la formación necesaria para fomentar una educación de calidad, acorde a los estándares establecidos a nivel mundial.

A nuestro juicio, la naturaleza misma de la educación debe fomentar procura de la calidad, a nivel de infraestructura, organización administrativa y curricular, recursos humanos y demás aspectos que 
confluyen para ofrecer una buena educación para los estudiantes. No es lo mismo, en efecto, un servicio educativo prestado en un centro privado que el mismo servicio prestado en uno público, ni es irrelevante que el personal sea funcionario $0 \quad$ contratado laboralmente, ni que se trate de centros unitarios o graduados, que impartan sólo educación infantil o toda la gama preuniversitaria, que sean plenamente formativos o se orienten a la capacitación en ciertas habilidades orientadas a la actividad profesional (academias). (Areche, V, 2016)

Los indicadores de calidad deben servir para dar mayor fuerza a los responsables educativos y a los docentes en la toma de decisiones y orientarlos hacia las prácticas educativas más adecuadas, porque finalmente, la clave de la calidad y la excelencia educativa se encuentra en las personas (Malpica, F, 2013) La norma es un modelo creado por la Organización Internacional de Normalización, también llamada Organización Internacional de Estandarización (originalmente en inglés: International Organization for Standardization, conocida por la abreviación ISO) es una organización para la creación de estándares internacionales compuesta por diversas organizaciones nacionales de normalización, para sistematizar los estándares que se requieren en sistemas de calidad en empresas de distintos tamaños siendo grandes, medianas o pequeñas empresas, privadas o públicas y de distinta actividad económica. Este sistema de gestión de calidad se cumple rigiéndose por medio de una disciplina para cumplir con una labor de excelencia para mejorar la calidad de un producto o servicio, tomando en cuenta la satisfacción del cliente.

\section{Materiales y métodos}

La investigación se enmarca en el enfoque cuantitativo de investigación, usando como diseño de la misma, siguiendo un tipo de investigación exploratoria y descriptiva que permitirá conocer la situación que presenta la institución que se estudia y determinar si es susceptible de aplicar las normas ISO 9001 -2015 para mejorar la calidad académica.

Para la recolección de la información se aplicaron las técnicas de encuesta y entrevista autoadministrados u la observación. 
Los instrumentos fueron entrevista autoadministrada, cuestionario online usando Google Drive y lista de chequeo para registrar la observación de documentos. Estos instrumentos se aplicaron a diferentes actores y en distintos momentos de la investigación. Los cuestionarios se aplicaron al personal docente y administrativo de la institución, la entrevista autoadministrada a los directivos de esta y la lista de cotejo para el análisis de los documentos consultados.

Los instrumentos pasaron el requerido procedo de validación por expertos para garantizar su rigor científico y los datos de entrevistas y análisis documental se hicieron atendiendo a las categorías definidas para tal fin y que se desprenden de los objetivos planteados. Para su análisis se usó la técnica simple de reducción de datos.

\section{Resultados}

Pregunta No 2. ¿Conoce usted cada uno de los procesos y documentos que requiere la institución educativa para realiza de forma eficaz y eficiente su trabajo?
Tabla 1. Resultados de pregunta 2 de encuesta realizada.

\begin{tabular}{|l|c|c|}
\hline Alternativas & Frecuencia & Porcentaje \\
\hline Mucho & 3 & $14 \%$ \\
\hline Algo & 17 & $77 \%$ \\
\hline Poco & 2 & $9 \%$ \\
\hline Nada & 0 & $0 \%$ \\
\hline Total & $\mathbf{2 2}$ & $\mathbf{1 0 0} \%$ \\
\hline
\end{tabular}

Elaborado por: Autora de tesis.

Gráfico 1. Resultados en gráfica de pregunta 2 de encuesta realizada.

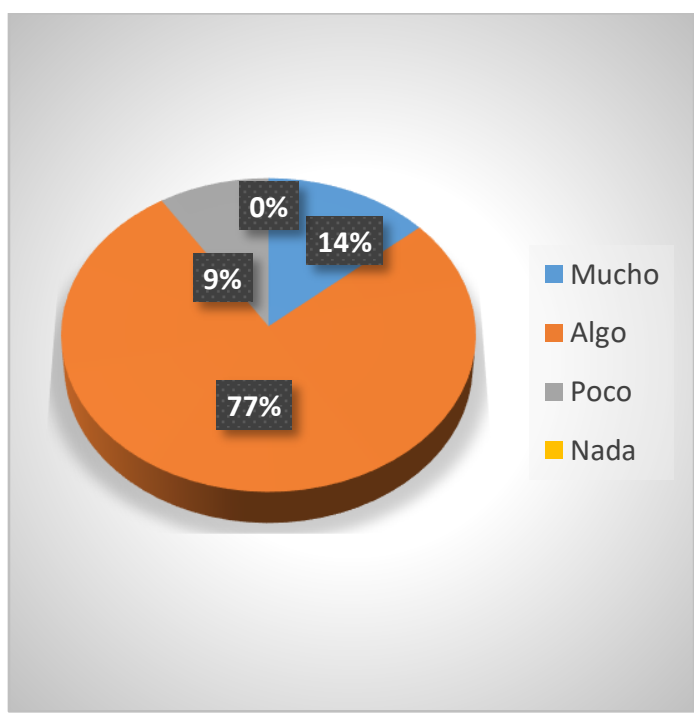

Fuente: Encuesta a talento humano. Elaborado por: Autora de tesis.

Análisis e interpretación de resultados

El $77 \%$ de los encuestados responden que tienen algo de conocimiento de los procesos y documentos que requiere la la institución educativa para realizar de forma eficaz y eficiente su trabajo, mientras que el $14 \%$ de ellos responde que mucho y el $9 \%$ que poco. 
Se establece que existe cierto nivel de desconocimiento en cuanto a procesos e información y documentos de parte del talento humano, los mismos que permitirían mejorar su actividad en beneficio de la excelencia académica.

Pregunta No 4. ¿Conoce usted qué es un sistema de Gestión de Calidad?

Tabla 2. Resultados de pregunta 2 de encuesta realizada.

\begin{tabular}{|l|c|c|}
\hline Alternativas & Frecuencia & Porcentaje \\
\hline Mucho & 5 & $23 \%$ \\
\hline Algo & 9 & $41 \%$ \\
\hline Poco & 8 & $36 \%$ \\
\hline Nada & 0 & $0 \%$ \\
\hline Total & $\mathbf{2 2}$ & $\mathbf{1 0 0} \%$ \\
\hline
\end{tabular}

Elaborado por: Autora de tesis.

Gráfico 2. Resultados en gráfica de pregunta 4 de encuesta realizada.

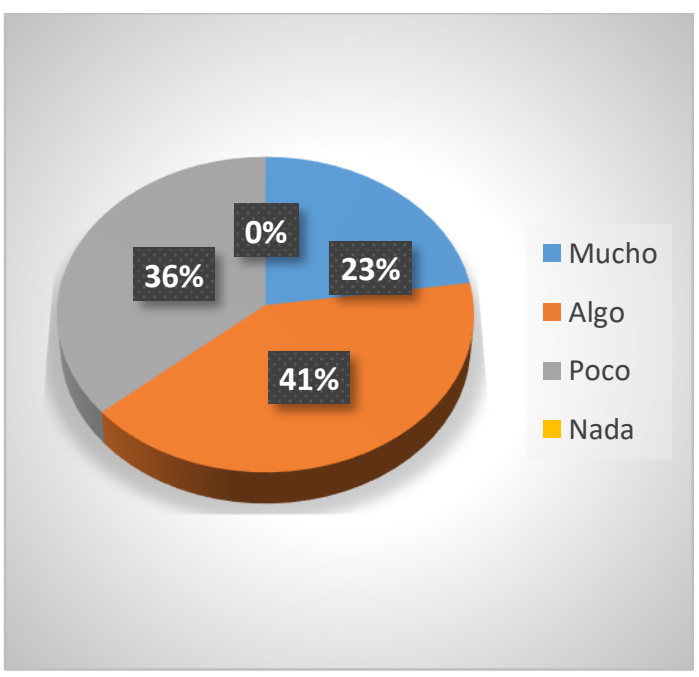

Fuente: Encuesta a talento humano. Elaborado por: Autora de tesis.
Análisis e interpretación de resultados

En esta pregunta el $44 \%$ de los encuestados responden que tienen algo de conocimiento acerca de lo que es un sistema de gestión de calidad, mientras que el $36 \%$ responde que tiene poco conocimiento y el $23 \%$ de ellos responde que mucho.

Se establece que existe desconocimiento acerca de sistemas de gestión de calidad por parte del personal, los mismos que permitirían mejorar su actividad en beneficio de la excelencia académica.

Pregunta No 5. ¿En la institución educativa se han implementado sistemas de gestión de calidad para mejorar la excelencia académica?

Tabla 3. Resultados de pregunta 5 de encuesta realizada.

\begin{tabular}{|l|c|c|}
\hline Alternativas & Frecuencia & Porcentaje \\
\hline Mucho & 0 & $0 \%$ \\
\hline Algo & 0 & $0 \%$ \\
\hline Poco & 3 & $14 \%$ \\
\hline Nada & 19 & $86 \%$ \\
\hline Total & $\mathbf{2 2}$ & $\mathbf{1 0 0} \%$ \\
\hline
\end{tabular}

Elaborado por: Autora de tesis. 
Gráfico 3. Resultados en gráfica de pregunta 5 de encuesta realizada.

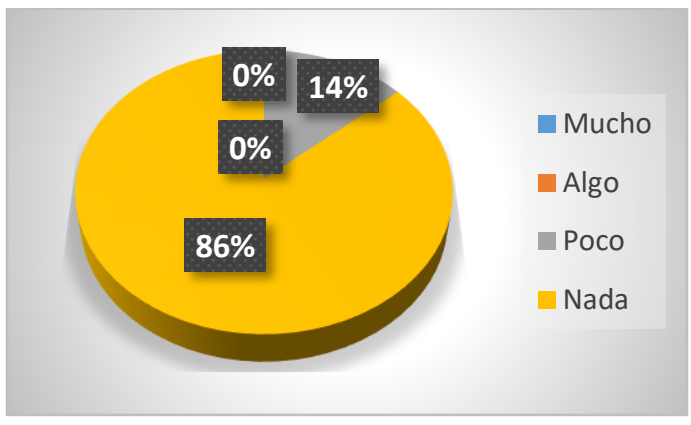

Fuente: Encuesta a talento humano. Elaborado por: Autora de tesis.

Análisis e interpretación de resultados

En esta pregunta el $86 \%$ de los encuestados responden que en la institución no se han implementado sistemas de gestión de calidad para mejorar la excelencia académica, el $14 \%$ responde que poco.

Este resultado permite constatar que en la institución no existe un sistema de gestión de calidad en aplicación.

\section{Pregunta No 9. ¿Considera} necesario implementar normas de calidad en la institución?

Tabla 4. Resultados de pregunta 9 de encuesta realizada.

\begin{tabular}{|l|c|c|}
\hline Alternativas & Frecuencia & Porcentaje \\
\hline Mucho & 17 & $77 \%$ \\
\hline Algo & 5 & $23 \%$ \\
\hline Poco & 0 & $0 \%$ \\
\hline Nada & 0 & $0 \%$ \\
\hline Total & $\mathbf{2 2}$ & $\mathbf{1 0 0} \%$ \\
\hline
\end{tabular}

Gráfico 4. Resultados en gráfica de pregunta 9 de encuesta realizada.

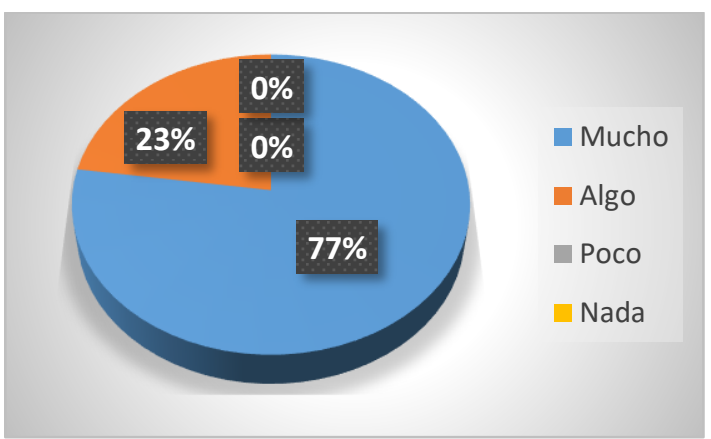

Fuente: Encuesta a talento humano. Elaborado por: Autora de tesis.

Análisis e interpretación de resultados

En esta pregunta, el $77 \%$ de los encuestados responden que es necesario implementar normas de calidad en la institución, el $23 \%$ de ellos manifiestan que algo.

Este resultado permite constatar la necesidad de implementar normas de calidad en la unidad educativa.

Pregunta No 12. ¿Qué percepción tiene usted de la calidad del servicio que brinda la institución?

Tabla 5. Resultados de pregunta 12 de encuesta realizada.

\begin{tabular}{|l|c|c|}
\hline Alternativas & Frecuencia & Porcentaje \\
\hline Excelente & 5 & $23 \%$ \\
\hline Buena & 11 & $50 \%$ \\
\hline Por mejorar & 6 & $27 \%$ \\
\hline Total & $\mathbf{2 2}$ & $\mathbf{1 0 0} \%$ \\
\hline
\end{tabular}

Elaborado por: Autora de tesis.

Elaborado por: Autora de tesis. 
Gráfico 5. Resultados en gráfica de pregunta 12 de encuesta realizada.

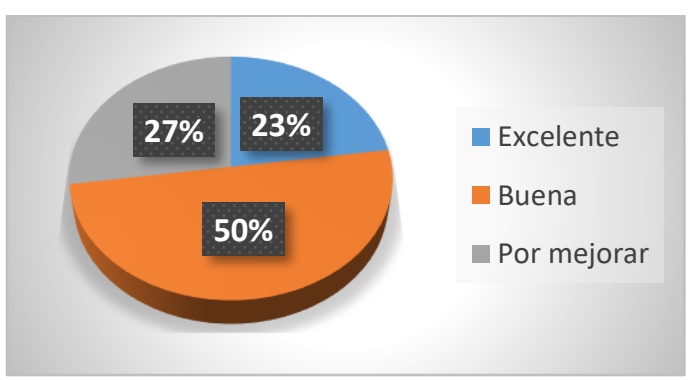

Fuente: Encuesta a talento humano. Elaborado por: Autora de tesis.

Análisis e interpretación de resultados

En cuanto a la percepción de la calidad del servicio que brinda la institución, el $50 \%$ de los encuestados responden que la consideran buena, el $27 \%$ responde que hay aspectos por mejorar, el $23 \%$ manifiesta que es excelente.

Este resultado permite constatar la percepción de calidad la misma que tiene aspectos por mejorar.

Pregunta No 13. ¿Tiene usted conocimiento acerca de la norma de calidad ISO 9001-2015 aplicada a instituciones educativas?

Tabla 6. Resultados de pregunta 13 de encuesta realizada.

\begin{tabular}{|l|c|c|}
\hline Alternativas & Frecuencia & Porcentaje \\
\hline Mucho & 2 & $9 \%$ \\
\hline Algo & 0 & $0 \%$ \\
\hline Poco & 1 & $5 \%$ \\
\hline Nada & 19 & $86 \%$ \\
\hline Total & $\mathbf{2 2}$ & $\mathbf{1 0 0} \%$ \\
\hline
\end{tabular}

Elaborado por: Autora de tesis.
Gráfico 6. Resultados en gráfica de pregunta 13 de encuesta realizada.

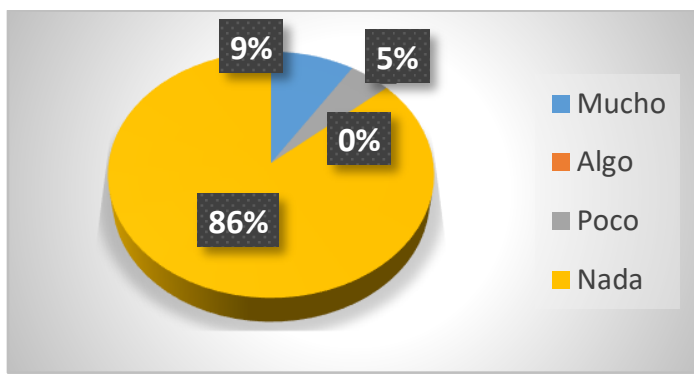

Fuente: Encuesta a talento humano. Elaborado por: Autora de tesis.

\section{Análisis e interpretación de resultados}

En cuanto al conocimiento acerca de la norma de calidad ISO 9001-2015 aplicada a instituciones educativas, el $86 \%$ de los encuestados responden que no tienen conocimiento alguno, el 9\% tiene mucho conocimiento y el $5 \%$ tiene poco conocimiento.

Este resultado permite constatar el desconocimiento sobre la norma de calidad ISO 9001-2015 aplicada a instituciones educativas.

Pregunta No 14. ¿Está usted dispuesto a colaborar y comprometerse con la implementación de un sistema de Gestión de Calidad en procura de brindar un servicio de excelencia a la comunidad? 
Tabla 7. Resultados de pregunta 14 de encuesta realizada.

\begin{tabular}{|l|c|c|}
\hline Alternativas & Frecuencia & Porcentaje \\
\hline Mucho & 21 & $95 \%$ \\
\hline Algo & 1 & $5 \%$ \\
\hline Poco & 0 & $0 \%$ \\
\hline Nada & 0 & $0 \%$ \\
\hline Total & $\mathbf{2 2}$ & $\mathbf{1 0 0} \%$ \\
\hline
\end{tabular}

Elaborado por: Autora de tesis.

Gráfico 7. Resultados en gráfica de pregunta 14 de encuesta realizada.

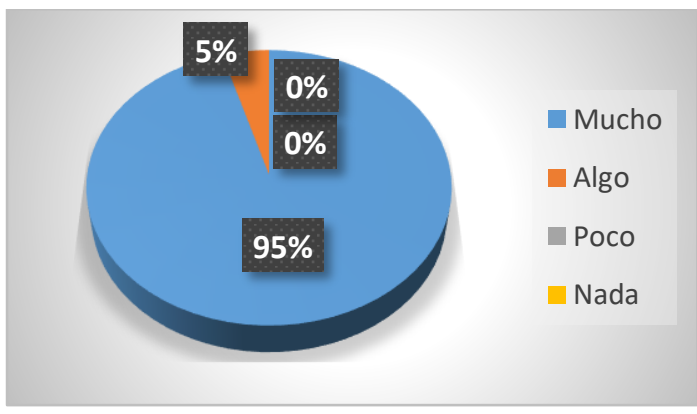

Fuente: Encuesta a talento humano. Elaborado por: Autora de tesis.

\section{Análisis e interpretación de} resultados

En cuanto al compromiso con la implementación de un Sistema de Gestión de Calidad en procura de brindar un servicio de excelencia a la comunidad, el $95 \%$ manifiesta el mismo, el $5 \%$ en menos nivel, pero lo acepta.

Se manifiesta el compromiso con la implementación de un Sistema de Gestión de Calidad en procura de brindar un servicio de excelencia a la comunidad por parte del talento humano institucional.

\section{Discusión}

En cuanto a la pregunta de si se tiene conocimiento acerca de cada uno de los procesos y documentos, el $77 \%$ (pregunta 3) de los encuestados manifiestan que tienen algo de conocimiento, lo cual es confirmado por la autoridad en la respectiva entrevista. El mismo porcentaje de encuestados manifiestan además que tienen poco conocimiento acerca de la misión, visión, objetivos y políticas institucionales planteadas por la unidad educativa lo cual incide en la falta de compromiso institucional de parte del personal, como lo dice la autoridad en la entrevista. De igual manera se desconoce, por parte del personal, la estructura orgánica institucional y sus respectivas funciones (pregunta $8 ; 86 \%)$

Debido a que en la institución educativa no se han implementado sistemas de gestión de calidad (pregunta 5; 86\%), el personal no tiene conocimiento sobre el tema (pregunta $4 ; 77 \%$ ), debido a ello no se realizan evaluaciones del desempeño docente (pregunta 6; $77 \%$ ). Por estos motivos el $77 \%$ de los encuestados consideran que es 
necesario implementar normas de calidad en la institución (pregunta 9) y así mejorar aspectos como: evaluación al personal, recursos tecnológicos, trabajo en equipo, capacitación del personal (pregunta 11) para así mejorar la percepción de la calidad de los servicios (pregunta 12 y entrevista).

Según la Norma ISO 9001:2015C, la adopción de un sistema de gestión de la calidad es una decisión estratégica para una organización que le puede ayudar a mejorar su desempeño global y proporcionar una base sólida para las iniciativas de desarrollo sostenible. Los beneficios potenciales de implementar un sistema de gestión de la calidad basado en esta norma internacional (ISO, 2015) son para una organización:

a. La capacidad para proporcionar regularmente productos y servicios que satisfagan los requisitos del cliente y los legales y reglamentarios aplicables;

b. Facilitar oportunidades de aumentar la satisfacción del cliente;

c. Abordar los riesgos y oportunidades asociadas con su contexto y objetivos; d. La capacidad de demostrar la conformidad con requisitos del sistema de gestión de calidad especificados

Con estos antecedentes se plantean los siguientes objetivos: Objetivo general, proponer el uso de un sistema de gestión de calidad basado en la norma ISO 9001:2015(C) como herramienta que contribuya a la mejora académica en el colegio Rubén Darío.

Esta investigación tuvo por objetivo el diseño de un modelo de calidad para el colegio Rubén Darío con la intención de mejorar su calidad educativa, teniendo como base la norma ISO 9001:2015(C).

La educación forma para que el individuo se aventure por sí mismo a explorar el mundo y construir el conocimiento que le permita entender las causas de las cosas y de los fenómenos y alcance la libertad necesaria frente al mundo, al aproximarse sucesivamente a la verdad (León, 2012).

Las instituciones educativas son organizaciones complejas en donde se conjugan muchos factores, cuyo manejo demanda el concurso de excelentes líderes que se ven enfrentados a manejar diferentes aspectos de la organización, 
generalmente no fáciles de articular (Cárdenas, 2017).

De acuerdo con Drummond (2001) en la prestación de un servicio, satisfacción del cliente significa cumplimiento de las expectativas. $Y$ cumplir las expectativas significa a su vez:

- Identificar los principales determinantes de la calidad;

- Manejar las expectativas del cliente;

- Impresionar al cliente;

- Instruir al cliente;

- Crear sistemas de apoyo fiables;

- Solicitar al cliente su opinión.

Para el caso del presente estudio se toma como referencia de cliente a los padres de familia como usuarios del servicio educativo pues son ellos quienes escogen la institución educativa y uno de los criterios que consideran es el de la calidad de esta.

La actual gestión está definida por el paso de un sistema "de antes" centralizado y jerárquico a un sistema "de ahora" con la reforma educativa en la cual se tiende hacia una descentralización a nivel distrital, con el propósito de llegar a un desarrollo del sistema educativo en el sector público. (Pacheco, 2018).

El objetivo de educar con calidad implica no solo que el profesor debe dominar un tema, y que para asumir la responsabilidad de ser tutor debe tener las cualidades personales y académicas idóneas, sino que las dinámicas de clases, de evaluaciones y de seguimiento de los alumnos deben garantizar tanto alcanzar los objetivos institucionales propuestos como llenar las expectativas que se han fijado los estudiantes, estableciendo indicadores de cumplimiento de metas (Díaz, T, 2009).

La norma ISO 9001:2015@ se sustenta con siete principios de gestión de calidad de las cuales fueron elaborados y renovados por especialistas internacionales, cuyo beneficio es relacionar estos principios con la norma para el éxito de los resultados y son los siguientes (ISO, 2015):

Enfoque al cliente: ganar la confianza del cliente, interactuar sobre sus necesidades actuales y futuras, fomenta un valor agregado hacia el consumo del cliente. 
Liderazgo: la alta dirección debe comprometer a todas las partes interesadas para que se alineen las estrategias desarrolladas con los objetivos de la organización.

Compromiso de las personas: involucrar a todo el personal de todos los niveles, valorar sus competencias, habilidades, y capacitarlos, aquello agrega valor a la organización.

\section{Enfoque basado en procesos:} entender toda la organización como procesos interrelacionados permitirá resultados eficaces, así como optimización en sus recursos y procesos.

Mejora: tener una consecución de mejora continua extenderá las oportunidades para que la empresa mantenga los resultados deseados con los cambios externos e internos que se den.

\section{Toma de decisiones basada en la} evidencia: escoger una decisión se debe sustentar con evidencias de documentos, datos, pruebas de análisis para comprender las causas y consecuencias de la toma de decisiones.

Gestión de las relaciones: sostener una buena relación con proveedores, socios y todas las partes interesadas que involucre a los procesos permitirá que la organización mantenga su éxito.

\section{Conclusiones}

Al finalizar la investigación se establecen las siguientes conclusiones:

En la Unidad Educativa Rubén Darío, se encontraron algunas deficiencias, como por ejemplo no cuenta con una política de calidad, ni objetivos referidos a ese aspecto, un organigrama y además existe reprocesos que afecta al área administrativa, una visión y misión difusas, además del desconocimiento de estos en el personal. Mediante el uso de las técnicas de investigación, se realizó encuesta al talento humano de la institución, con la finalidad de realizar un estudio referente a la importancia de la implementación de un sistema de gestión de calidad.

Se afirma que el sistema de calidad ISO 9001:2015@ funciona y sirve como una herramienta de gestión que orienta al alcance de la excelencia académica en la Unidad Educativa Rubén Darío. Se concluye que la norma ISO 9001:2015C, la 
cual propone una serie de requisitos que ayuda a la gestión del colegio, la organización del trabajo, la eficiencia en los procesos, el liderazgo, la mejora continua se puede obtener como resultado la mejora en la calidad del servicio educativo que conlleva a un nivel de satisfacción óptimo por parte de los usuarios.

El personal de la institución manifiesta su compromiso para el caso de que se implemente un sistema de gestión de calidad.

\section{Bibliografía}

Araujo, A. (2016). Sistema de gestión de calidad basado en la norma ISO 9001 para ser aplicado en una institución educativa de Guayaquil. Obtenido de http://repositorio.ug.edu.ec/bit stream/redug/31521/1/Tesis \%20Alan\%20\%20Araujo\%20 Ballestero.pdf

Areche, V. (2013). La gestión institucional y la calidad en el servicio educativo según la percepción de los docentes y padres de familia del 3ro, 4to y 5to de Secundaria del colegio María Auxiliadora de Huamanga - Ayacucho 2011. http://cybertesis.unmsm.edu. pe/handle/cybert.

Aristizabal, G. (2009). Impacto de la certificación ISO 9001 en la visión y misión de una institución educativa. Obtenido de https://www.javeriana.edu.co/ biblos/tesis/educacion/tesis88 .pdf

Burckhardt, V. (2016). Estrategia y desarrollo de una guía de implantación de la norma ISO 9001-2015.

España: Valenciana.

Cárdenas, C. (2017). ¿Existe relación entre la gestión administrativa y la gestión educativa? Obtenido de file://C:/Users/user/Download s/Dialnet-

ExisteRelacionEntreLaGestio nAdministrativaYLalnnov5800411.pdf

Díaz, T. (2009). Excelencia académica y formación integral. Centro Editorial Universidad del Rosario. I edición.

Drummond, H. (2001). La calidad total. Deusto.

ISO. (2015). Norma ISO 9001-2015. https://www.iso.org/obp/ui/\#is o:std:iso:9001:ed-5:v1:es.

León. (2012). Los fines de la educación.

https://www.redalyc.org/pdf/7 09/70925416001.pdf.

López, F. (2003). La gestión de la calidad en la educación. La Muralla. 3era edición.

López, P. (2010). Variables asociadas a la gestiòn escolar 
como factores de calidad educativa. Obtenido de https://scielo.conicyt.cl/scielo. php?script $=$ sci_arttext\&pid $=S$ 0718-07052010000100008

Malpica, F. (2013). 8 Ideas clave. Calidad de la práctica educativa. Editorial GRAO, 1a Edición.

Ortiz, O. (2016). Sistema de gestión de calidad. Teoría y práctica bajo la norma ISO 9001.2015. ECOE ediciones.

Pacheco, R. (2018). Análisis de la gestión administrativa en las instituciones educativas. Obtenido de https://scielo.conicyt.cl/scielo. php?script=sci_arttext\&pid=S 0718-07642018000500259

Pérez, R. (2000). Hacia una educación de calidad: gestión, instrumentos y evaluación. Ediciones Narcea, 3aㅗ edición.

Ribera, J. (2007). Calidad: definirla, medirla y gestionarla. Ediciones Folio. 